\title{
Highly Optimized Iron Oxide Embedded Poly (Lactic Acid) Nanocomposites for Effective Magnetic Hyperthermia and Biosecurity
}

\author{
Chiseon Ryu' \\ Hwangjae Lee' \\ Hohyeon $\mathrm{Kim}^{2}$ \\ Seong Hwang' \\ Yaser Hadadian (1D) ${ }^{2,3}$ \\ Ayeskanta Mohanty ${ }^{4}$ \\ In-Kyu Park (D) ${ }^{4}$ \\ Beongki Cho' \\ Jungwon Yoon ${ }^{2,3}$ \\ Jae Young Lee (D) ${ }^{1}$
}

'School of Materials Science and Engineering, Gwangju Institute of Science and Technology, Gwangju, 61005,

Republic of Korea; ${ }^{2}$ School of Integrated

Technology, Gwangju Institute of Science and Technology, Gwangju, 61005,

Republic of Korea; ${ }^{3}$ Research Center for Nanorobotics in Brain, Gwangju Institute of Science and Technology, Gwangju, 61005, Republic of Korea; ${ }^{4}$ Department of Biomedical Sciences, Chonnam National University Medical School, Hwasun-gun, Jeollanam-do, Republic of Korea
Correspondence: Jungwon Yoon; Jae Young Lee

Tel +82 62715 5332; +82 627152358

Email jyoon@gist.ac.kr;

jaeyounglee@gist.ac.kr
Introduction: Iron oxide magnetic nanoparticles (IONPs) have attracted considerable attention for various biomedical applications owing to their ease of synthesis, strong magnetic properties, and biocompatibility. In particular, IONPs can generate heat under an alternating magnetic field, the effects of which have been extensively studied for magnetic hyperthermia therapy. However, the development of IONPs with high heating efficiency, biocompatibility, and colloidal stability in physiological environments is still required for their safe and effective application in biomedical fields.

Methods: We synthesized magnetic IONP/polymer nanocomposites (MNCs) by embedding IONPs in a poly(L-lactic acid) (PLA) matrix via nanoemulsion. The IONP contents (Fe: 9$22[\mathrm{w} / \mathrm{w}] \%$ ) in MNCs were varied to investigate their effects on the magnetic and hyperthermia performances based on their optimal interparticle interactions. Further, we explored the stability, cytocompatibility, biodistribution, and in vivo tissue compatibility of the MNCs.

Results: The MNCs showed enhanced heating efficiency with over two-fold increase compared to nonembedded bare IONPs. The relationship between the IONP content and heating performance in MNCs was nonmonotonous. The highest heating performance was obtained from $\mathrm{MNC} 2$, which contain $13 \% \mathrm{Fe}(\mathrm{w} / \mathrm{w})$, implying that interparticle interactions in MNCs can be optimized to achieve high heating performance. In addition, the MNCs exhibited good colloidal stability under physiological conditions and maintained their heating efficiency during $48 \mathrm{~h}$ of incubation in cell culture medium. Both in vitro and in vivo studies revealed excellent biocompatibility of the MNC.

Conclusion: Our nanocomposites, comprising biocompatible IONPs and PLA, display improved heating efficiency, good colloidal stability, and cytocompatibility, and thus will be beneficial for diverse biomedical applications, including magnetic hyperthermia for cancer treatment.

Keywords: iron oxide nanoparticle, hyperthermia, inter-particle interactions, nanomedicine

\section{Introduction}

Iron oxide nanoparticles (IONPs) have garnered significant attention owing to their strong magnetization, ease of synthesis, biocompatibility, and in vivo biodegradability. ${ }^{1-3}$ Interestingly, they generate heat in an alternating magnetic field (AMF), which allows remote heat generation using IONPs and AMF, even in deep tissue. ${ }^{4}$ Therefore, AMF-induced heating systems has been widely used for various biomedical applications, such as hyperthermia cancer treatment, ${ }^{5-7}$ drug delivery, ${ }^{8-10}$ and deep brain stimulation. ${ }^{11-13}$ Colloidal magnetic nanoparticles, including IONPs, collectively generate heat in the surrounding medium. The 


\section{Graphical Abstract}
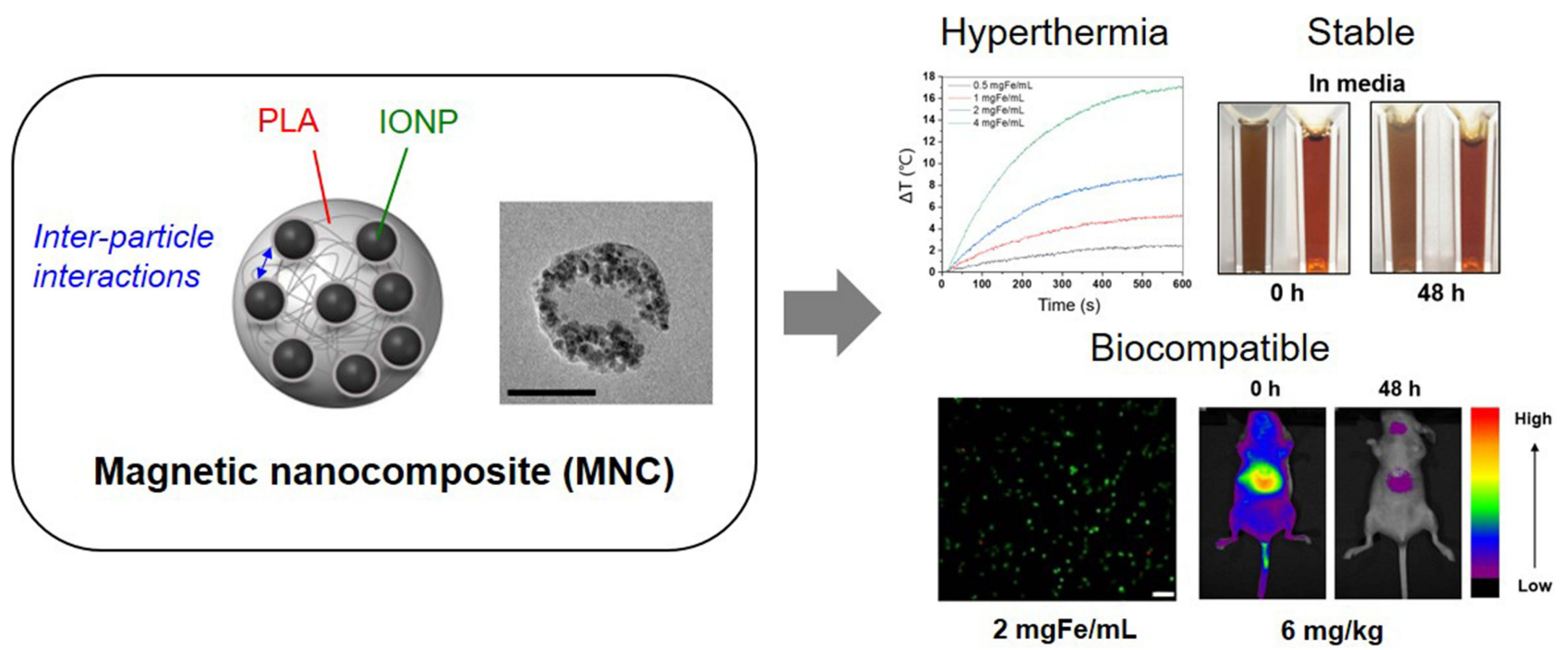

magnetic and heat generation properties of IONPs are highly influenced by multiple factors, such as composition, size, shape, and interparticle interactions. ${ }^{14-16}$ These individual parameters have been experimentally and theoretically studied to improve the heat generation efficiency of IONPs. Therefore, small amounts of IONPs with low magnetic fields are favored to avoid their potential toxicity in various biomedical applications. ${ }^{17-19}$ Hence, extensive efforts have been made to synthesize IONPs with high heat generation efficiency. ${ }^{20-22}$ IONPs mainly generate heat in response to AMF via relaxation loss and/or hysteresis losses. Superparamagnetic singledomain magnetic nanoparticles at a low concentration in an AMF typically generate heat via Néel relaxation and Brownian relaxation. ${ }^{23,24}$ In addition, interparticle interactions between IONPs can play dominant roles in the overall magnetic and hyperthermia properties of interacting IONPs (eg, clusters and concentrated ferrofluids). ${ }^{25-27}$ The close proximity of multiple IONPs can affect the local dipole and anisotropic energy, resulting in an alteration of their magnetic susceptibility and heating efficiency. ${ }^{28-30}$ Hence, several attempts have been made to modulate the interparticle interactions of IONPs to enhance the magnetic or hyperthermia characteristics. Several studies have reported that interparticle interactions (dipole-dipole interactions) of IONPs can increase or decrease magnetic relaxation events and heat conversion, for which clear mechanisms have not been suggested. ${ }^{31-33}$ Nevertheless, it has been reported that optimization of interparticle interactions (such as spacing and density) can enhance the overall heating efficiencies of various magnetic nanoparticle clusters and ensembles. ${ }^{26,27,34}$ Evans et al investigated the effects of magnetic nanoparticle (magnetite) concentrations in silicone composites on heat generation. ${ }^{35}$ They found that heat generation was maximal at $1[\mathrm{v} / \mathrm{v}] \%$ magnetic nanoparticle concentration, and the optimal concentration was affected by the applied magnetic field. In addition, the colloidal stability of MNPs in biological environments is pivotal because MNPs readily aggregate in vivo and lead to substantial problems, such as reduction of heating performance, cytotoxicity, and thrombosis. ${ }^{36-38}$ Altogether, it is highly desirable to develop MNPs with high heat generation efficiency and colloidal stability in physiological environments for effective and biocompatible applications of MNPs. ${ }^{39,40}$ In this study, we produced IONPs/poly lactic acid (PLA) magnetic nanocomposites (MNCs) by incorporating different amounts of IONPs into the PLA matrix, in which the IONPs within the solid polymeric matrices were expected to have different interparticle distances (interactions) depending on their contents. Specifically, we synthesized hydrophobic IONPs (oleic acid-coated IONPs) and embedded them in PLA using an oil-in-water emulsion while varying the contents of IONPs and PLA (Scheme 1). We hypothesized that specific IONP content in the PLA matrix could 


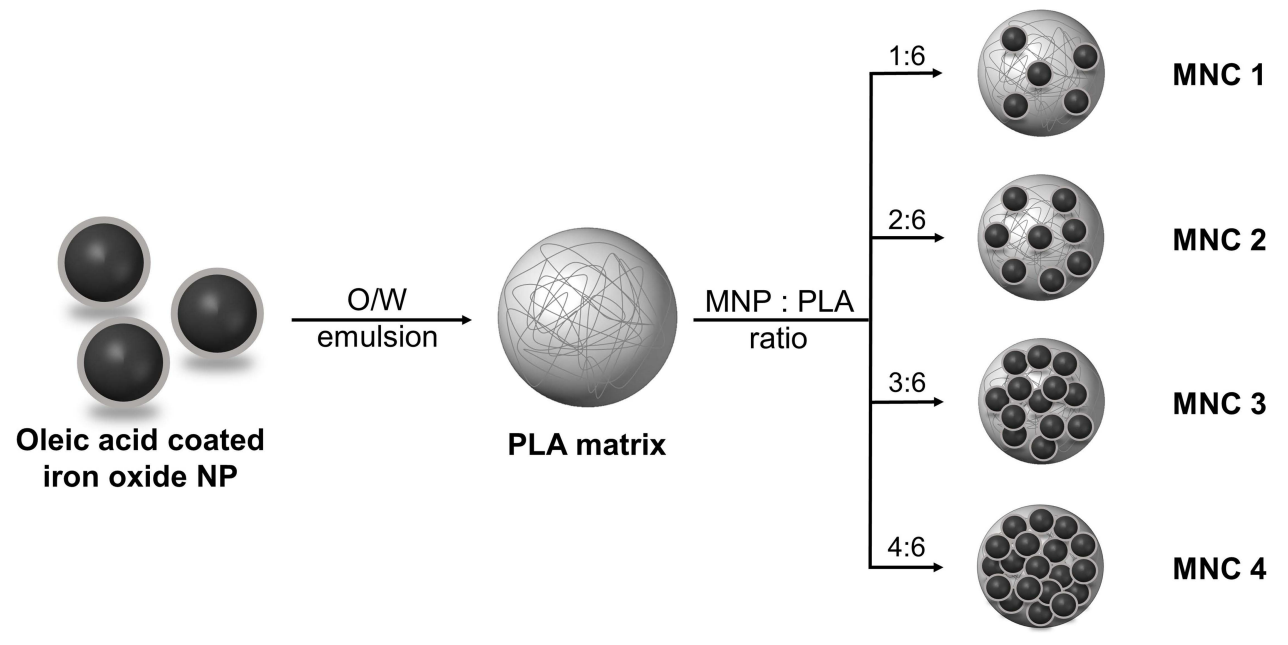

Scheme I Schematic for the synthesis of iron oxide nanoparticles (IONPs)-embedded poly(lactic acid) (PLA) magnetic nanocomposites (MNCs) via oil-in-water emulsion by differing the contents of IONPs in the MNCs.

enhance efficient hyperthermia properties via optimized interparticle interactions. Furthermore, PLA-based IONP encapsulation (ie, MNCs) was expected to offer high stability and biocompatibility in biological environments. Notably, PLA is a biocompatible and biodegradable synthetic polymer that has been extensively used for various biomedical applications. ${ }^{41}$ Hence, given that the main components (ie, PLA and IONPs) are generally recognized as biocompatible, our MNCs could be expected to be biocompatible. Our IONP/polymer nanocomposite system may provide insights for improving the heating efficiency and stability of other magnetic nanoparticles by simply controlling the particle content in biocompatible polymer matrix.

\section{Materials and Methods Synthesis of Oleic Acid-Coated Iron Oxide Nanoparticles}

Oleic acid-coated IONPs were synthesized according to a previously reported co-precipitation method. ${ }^{42}$ Briefly, iron (II) chloride tetrahydrate $\left(\mathrm{FeCl}_{2} \cdot 4 \mathrm{H}_{2} \mathrm{O}\right)(4.3 \mathrm{~g})$, and Iron (III) chloride hexahydrate $\left(\mathrm{FeCl}_{3} \cdot 6 \mathrm{H}_{2} \mathrm{O}\right)(11.6 \mathrm{~g})$ were completely dissolved in deionized water (DIW) $(350 \mathrm{~mL})$ in a three-neck round-bottom flask. The flask was then purged with nitrogen gas $\left(\mathrm{N}_{2}\right)$ and heated to 80 ${ }^{\circ} \mathrm{C}$ with vigorous stirring. Next, ammonium hydroxide solution $\left(\mathrm{NH}_{4} \mathrm{OH}, \sim 25 \mathrm{wt} \%\right)(20 \mathrm{~mL})$ was added, and the mixture was stirred for $5 \mathrm{~min}$. Subsequently, oleic acid solution ( $3 \mathrm{~mL}$ ) was added to the flask and then stirred for an additional $25 \mathrm{~min}$. The mixture was cooled to room temperature, and a black precipitate settled at the bottom. The black precipitate was washed three times with a mixture of DIW and ethanol $(1: 1, \mathrm{v} / \mathrm{v})$ to remove excess oleic acid and subsequently dried in an oven at $60{ }^{\circ} \mathrm{C}$. $\mathrm{FeCl}_{2} \cdot 4 \mathrm{H}_{2} \mathrm{O}, \mathrm{FeCl}_{3} \cdot 6 \mathrm{H}_{2} \mathrm{O}, \mathrm{NH}_{4} \mathrm{OH}$, and oleic acid were purchased from Sigma-Aldrich, USA.

\section{Synthesis of Magnetic Nanocomposites}

The MNCs were prepared based on the modified methods described in the literature. ${ }^{41}$ Briefly, the oleic acid-coated IONPs $(30,60,90$, or $120 \mathrm{mg})$ were first suspended in chloroform (7.5 mL). Then, $180 \mathrm{mg}$ of poly(L-lactide) (PLA; 260 kDa, Sigma-Aldrich) was added and dissolved in IONP-containing solution. Next, $36 \mathrm{~mL}$ of $8 \%(\mathrm{w} / \mathrm{v})$ polyvinyl alcohol (PVA; 30-70 kDa, Sigma-Aldrich) was added and sonicated for $3 \mathrm{~min}$ to form an oil/water emulsion, which was then stirred for $24 \mathrm{~h}$ to evaporate the chloroform. The solution was subsequently centrifuged at $17,000 \mathrm{rpm}$ for $30 \mathrm{~min}$. The particles were collected and dispersed in DIW. The samples were washed three times by centrifugation, resuspended in DIW, and finally stored at $4{ }^{\circ} \mathrm{C}$ for further use. The individual MNCs synthesized with IONPs of 30, 60, 90, and $120 \mathrm{mg}$ during emulsion were denoted as MNC1, MNC2, MNC3, and MNC4, respectively.

\section{Iron Content Quantification}

The iron content of each MNC was quantified using a colorimetric method. A colorimetric standard was first plotted using $\mathrm{FeCl}_{3} \cdot 6 \mathrm{H}_{2} \mathrm{O}$ and $\mathrm{FeCl}_{2} \cdot 4 \mathrm{H}_{2} \mathrm{O}$ (2:1, w/w). Briefly, various concentrations of $\mathrm{Fe}$ were prepared in 
hydrochloric acid (HCl, Duksan, Republic of Korea) solution, and $50 \mu \mathrm{L}$ of each solution was transferred into a 96well plate. Subsequently, $50 \mu \mathrm{L}$ of $1 \%$ ammonium phosphate (AP, Sigma-Aldrich) and $100 \mu \mathrm{L}$ of $0.1 \mathrm{M}$ potassium thiocyanate (KSCN, Sigma-Aldrich) were added to the solution. After 5 min of reaction, the absorbance of the solution was measured at $490 \mathrm{~nm}$ using a microplate reader. The MNC sample was first lyophilized. Subsequently, the dehydrated sample was dissolved in $\mathrm{HCl}$ and reacted with the reagents using the same procedures as the standard samples. Finally, the iron content of each sample was calculated using the standard curve.

\section{Materials Characterization}

The morphologies and shapes of the synthesized nanomaterials were characterized using transmission electron microscopy (TEM, Technai G2S Twin) at $300 \mathrm{keV}$. Dynamic light scattering and zeta potential measurements were performed using a zeta-potential and particle-size analyzer (ELS Z2 Plus; Photal Otsuka Electronics). The crystal structure of the IONPs was analyzed by X-ray diffraction (XRD, Rigaku SmartLab). The magnetic properties of the particles were measured using a vibrating sample magnetometer (VSM, Lake Shore Cryotronics 7400 series) in a magnetic field ranging from $-9 \mathrm{kOe}$ to 9 kOe. From the hysteresis loop of each sample, a hysteresis area was obtained for comparison. ${ }^{43}$

\section{Hyperthermia Test}

Magnetic heat generation was studied using IONPs, MNCs, and Resovist ${ }^{\circledR}$ (Meito Sangyo Co., Ltd. Nagoya, Japan). Solutions containing different concentrations ( 0.5 , 1, 2, and $4 \mathrm{mg} \mathrm{Fe} / \mathrm{mL}$ ) were prepared for each sample. Before the hyperthermia experiment, the initial temperature of the sample solution was maintained at $25^{\circ} \mathrm{C}$ using a water-circulating jacket. An AMF (OSH-120-B; Osung Hitech, Republic of Korea) with an amplitude ( $H, 3.25 \mathrm{kA} /$ $\mathrm{m})$ and frequency $(f, 191 \mathrm{kHz})$ was applied for $10 \mathrm{~min}$. In addition, to study the effects of the AMF conditions (ie, amplitude and frequency) on the heat generation of the prepared MNCs, a lab-made AMF (GIST) was employed. ${ }^{44}$ During AMF application, the temperature was monitored using an IR laser camera (FLIR SC300series). The heat generation efficiency of the magnetic nanoparticles, which is typically referred to as specific loss power (SLP), was calculated using the following formula: ${ }^{6}$

$$
\text { Specific Loss Power(SLP })=\frac{c}{m_{F e}} \times\left.\frac{d T}{d t}\right|_{t \rightarrow 0}
$$

where $C$ is the specific heat capacity of water, $m_{F e}$ is the weight of iron, $d T$ is the temperature change, and $d t$ is the change in time.

\section{Stability Test}

For the stability tests, we performed both size analysis and hyperthermia analysis after incubating the samples in various solutions. First, MNC2 and Resovist ${ }^{\circledR}$ were incubated in PBS and phenol red-free cell culture medium (DMEM). The hydrodynamic diameters of individual samples were measured by DLS during incubation at $0 \mathrm{~h}, 6 \mathrm{~h}, 12 \mathrm{~h}, 24 \mathrm{~h}$, and $48 \mathrm{~h}$. SLP changes in the samples were measured after incubation in DMEM. MNC2 and Resovist ${ }^{\circledR}$ samples were diluted to $1 \mathrm{mg} \mathrm{Fe} / \mathrm{mL}$. The SLP value of each sample was obtained with AMF application for $10 \mathrm{~min}$ at $0 \mathrm{~h}$ and 48 $\mathrm{h}$ after incubation at $20^{\circ} \mathrm{C}$.

\section{In vitro Cytocompatibility Test}

The cytocompatibility of MNC2 and Resovist ${ }^{\circledR}$ was tested by in vitro NIH-3T3 (Korean Cell Line Bank) cell culture using a live/dead assay. The cells were seeded at a density of $2 \times 10^{4}$ cells/well in a 24-well plate and cultured in the Dulbecco's modified Eagle's medium (DMEM) for $12 \mathrm{~h}$. DMEM was supplemented with $10 \%$ fetal bovine serum (FBS) and 1\% antibiotic-antimycotic. Subsequently, the culture medium $(1 \mathrm{~mL})$ was replaced with the sample solution containing various concentrations of MNC2 or Resovist ${ }^{\circledR}$ in the culture medium and then incubated for an additional $24 \mathrm{~h}$. Cells with no particles were used as a positive control. After $24 \mathrm{~h}$, the sample solution was removed and washed with Dulbecco's phosphate-buffered saline (DPBS). For live/dead staining of the cells, the sample was stained with a staining solution comprising $1 \mu \mathrm{M}$ calcein-AM and $2 \mu \mathrm{M}$ ethidium homodimer-1 (EthD-1) in DPBS. After 5 min of incubation, the samples were washed with fresh DPBS. Fluorescent images of the cells were acquired using a fluorescence microscope (DMI3000B; Leica, Germany). At least nine images from each sample were randomly selected for cytotoxicity analysis. Green- and red-stained cells were counted as live and dead cells, respectively. Cell viability was calculated as a percentage of the number of live cells from the total number of cells (live + dead cells). Experiments were conducted in triplicate $(n=3)$. 


\section{In vivo Biodistribution and Toxicity Study}

All animal experiments were performed in accordance with the permission of the Institutional Animal Care and Use Committee (IACUC) of Chonnam National University, Republic of Korea (approval number: CNU IACUC-H-2020-25). Five-week-old male BALB/C nude mice (Orient Bio Inc., Korea) were anesthetized by vaporized isoflurane (Terrell; Piramal Critical Care Inc.) using a matrix vip 3000 vaporizer (Midmark Co.). For the biodistribution study, Cyanine 7 fluorescence dye (Cy 7) was loaded in MNC during $\mathrm{O} / \mathrm{W}$ emulsion. In brief, $800 \mu \mathrm{g}$ of the dye was added in oil phase while the other nanoemulsion procedure was the same as described above. The Cy7-loaded MNC was intravenously injected via tail vein with $6 \mathrm{mg} / \mathrm{kg}$ dose. In vivo fluorescence images of the animals were acquired at $0,4,8,12,24$, and $48 \mathrm{~h}$ after injection using FOBI (Fluorescence optical bio-imaging). Main organs (heart, liver, spleen, lung, and kidney) were harvested and their fluorescence images were acquired to compare MNC biodistribution. For histological evaluation of potential organ toxicity of the MNC, the mice were sacrificed $24 \mathrm{~h}$ and $48 \mathrm{~h}$ after injection. The heart, liver, spleen, lung, and kidney were harvested and fixed in $4 \%$ formaldehyde. Untreated mice were also used as the control (sham) for comparison. Samples were stained with hematoxylin and eosin (H\&E) according to the literature. ${ }^{45}$ Individual images were acquired by optical microscope (DMI3000B; Leica, Germany).

\section{Statistical Analysis}

The results are indicated as the mean \pm standard deviation (SD) unless otherwise noted. The differences among samples were compared by one-way analysis of variance (ANOVA) with Tukey's post hoc comparison at a significance level of 0.05 .

\section{Results and Discussion Synthesis of Magnetic Nanocomposites}

To synthesize MNCs, oleic acid-capped hydrophobic IONPs were first synthesized by the co-precipitation method. TEM images showed that the prepared IONPs had sphere-like shapes with an average diameter of $9.3 \pm$ $2.2 \mathrm{~nm}$ (Figure 1A). DLS measurements indicated that the average diameter of the particles was approximately 32.8 $\mathrm{nm}$ in chloroform (Figure 1B). Also, X-ray diffraction (XRD) pattern of the IONPs indicated the typical diffraction peaks for oleic-acid coated $\mathrm{Fe}_{3} \mathrm{O}_{4}$ nanoparticles
(Figure S1). The synthesized IONPs showed superparamagnetic behavior and a saturation magnetization of 56 $\mathrm{emu} / \mathrm{gFe}$ at $300 \mathrm{~K}$ (Figure 1C). Application of AMF to IONPs caused a dose-dependent temperature increase (Figure 1D). The SLP value of these oleic acid-capped IONPs was determined to be $37.2-41.6 \mathrm{~W} / \mathrm{gFe}$ in the concentration range of $0.5 \sim 4 \mathrm{mgFe} / \mathrm{mL}$ in the media (1octanol) (Figure S2).

MNCs containing different amounts of IONPs were prepared using oleic acid-coated IONPs and PLA in an oil/water single emulsion, in which the IONPs were expected to be firmly immobilized within the solid PLA polymer matrix (Scheme 1). The initial weight ratios of the IONPs and PLA were varied to $1: 6,2: 6,3: 6$, and 4:6, which resulted in the production of various MNCs with different local IONP densities and thus different interparticle interactions. MNC1, MNC2, MNC3, and MNC4 had $\mathrm{Fe}$ contents of 9, 13, 17, and $22 \mathrm{wt} \%$ ( $g \mathrm{Fe}$ per $g$ nanocomposites), respectively (Table 1 ). The average diameter of all MNCs was approximately $160 \mathrm{~nm}$ when measured by TEM, and their hydrodynamic diameter was approximately $170 \mathrm{~nm}$, as measured by DLS (Figure 2A and B). The zeta potential of MNCs was about $-15 \mathrm{mV}$, which was similar to PLA NPs (Figure S3). Consequently, we successfully obtained various MNCs with different IONP contents but similar overall sizes and distributions. Hence, we could further study the effects of IONP density and interparticle interactions within the solid PLA matrix on the magnetic and hyperthermia properties. Notably, this synthetic process can be further useful for effectively embedding hydrophobic molecules together with IONPs in a polymer matrix in a reproducible manner (Figure 2C). ${ }^{41}$ The Nomenclatures, compositions, and dimension of various MNCs are listed in Table 1.

\section{Magnetic Properties of Magnetic Nanomaterials}

The field-dependent magnetization of the various samples was measured using a VSM (Figure 3). Both IONPs and MNCs exhibited superparamagnetic-like behavior (Figure 3A). The saturation magnetization $\left(\mathrm{M}_{\mathrm{s}}\right)$ values of all the MNCs were higher than those of the nonencapsulated IONPs. The $\mathrm{M}_{\mathrm{s}}$ values of the bare IONPs, MNC1, $\mathrm{MNC} 2, \mathrm{MNC} 3$, and MNC4 were 56.6, 72.8, 77.5, 75.8, and 65.1 , emu $/ \mathrm{gFe}$ at $300 \mathrm{~K}$, respectively (Figure 3B). As the IONP content in the MNCs increased (to Fe 13\%), $\mathrm{M}_{\mathrm{s}}$ increased. Above this content, the $\mathrm{M}_{\mathrm{s}}$ values of the MNCs 
A

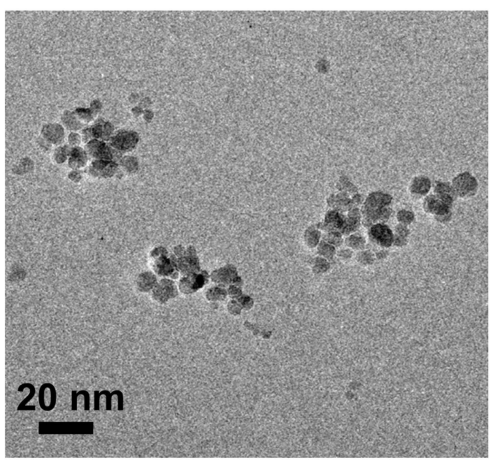

C

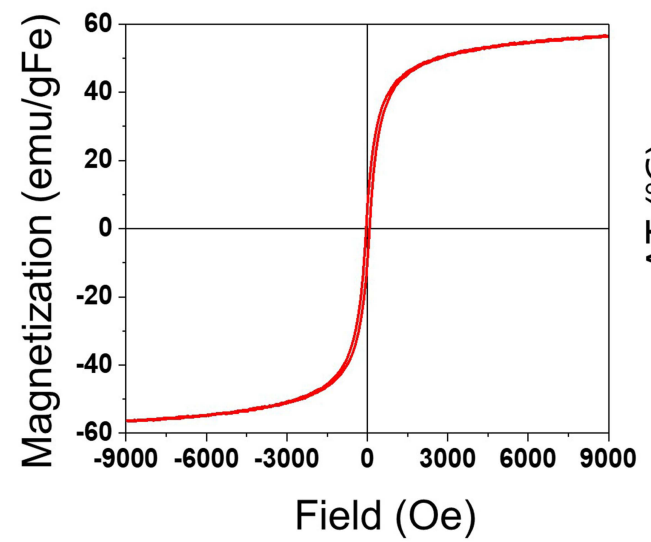

B
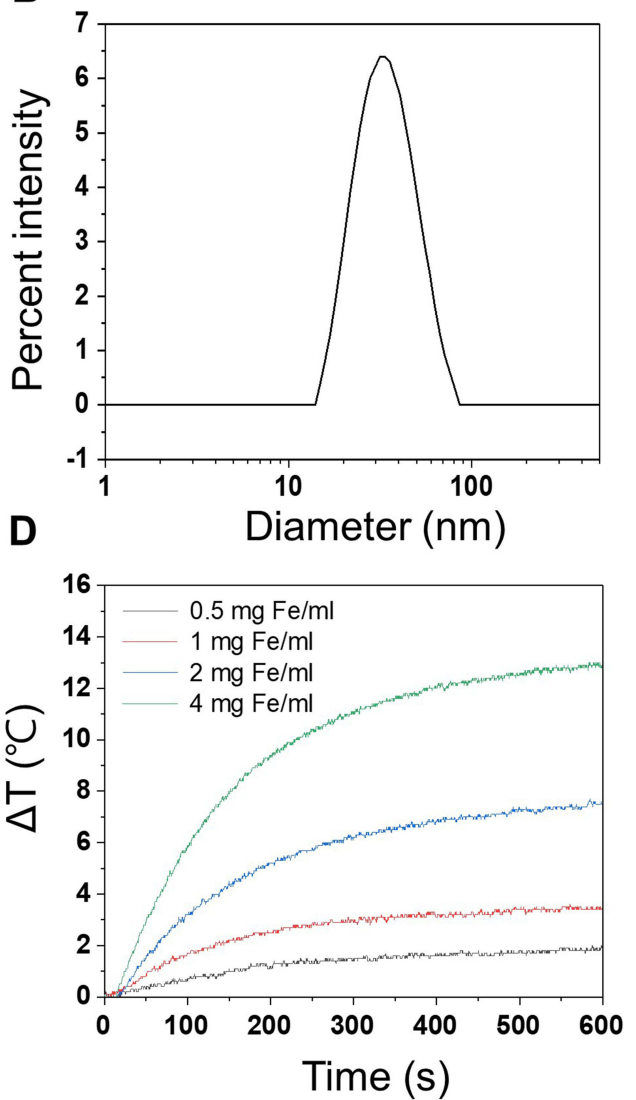

Figure I Characterization of the synthesized oleic acid-coated IONPs. (A) A TEM image of the IONPs. (B) A size distribution of the IONPs measured by DLS. (C) Magnetization curve of the IONPs. (D) Temperature increases of the sample solutions containing various amounts of IONPs under the AMF $(H=3.25 \mathrm{kA} / \mathrm{m}$ and $f=|9|$ $\mathrm{kHz})$.

decreased. As results, MNC2 had the highest $\mathrm{M}_{\mathrm{s}}$ value. The magnetic behavior of IONPs and their complexes can be explained as a combination of the intrinsic magnetic properties of single IONPs (eg, susceptibility) and their interparticle interactions (dipole-dipole interactions). For IONPs with short particle distances, the magnetic dipolar interactions can affect the effective anisotropy and relaxation behaviors of individual particles. Such changes can enhance or reduce the strength of magnetization. ${ }^{46-48}$ For example, IONP clusters can show increased net local magnetization and enhanced magnetic relaxivity. ${ }^{26}$ The dipolar coupling between closely packed IONPs can alter the anisotropic energy of the complex. ${ }^{49-52}$ For our MNCs, the interparticle interactions (IONPs embedded in PLA) appear to positively contribute to the magnetization at moderate particle concentrations. Conversely, in MNCs containing large amounts of IONPs (MNC3 and MNC4), excessive interparticle interactions appeared to reduce the magnetic properties, likely due to the reduction of the global magnetic response of the IONPs. We compared the hysteresis loss areas, which are known to be significantly correlated with interparticle interactions and hyperthermia efficiency. ${ }^{30}$ MNC1 and $\mathrm{MNC} 2$ samples had two times larger hysteresis loss areas than the other

Table I The Nomenclatures, Compositions, and Dimension of Various MNCs

\begin{tabular}{|l|c|c|c|c|c|}
\hline Sample Name & Input Weight Ratio (IONP:PLA) & Fe Content in MNC (wt\%) & Size by TEM (nm) & Size by DLS (nm) & PDI \\
\hline MNC I & $1: 6$ & 9 & 157.2 & 165.3 & 0.088 \\
MNC 2 & $2: 6$ & 13 & 160.4 & 167.8 \\
MNC 3 & $3: 6$ & 17 & 156.7 & 0.082 \\
MNC 4 & $4: 6$ & 22 & 161.9 & 170 & 0.051 \\
\hline
\end{tabular}


A
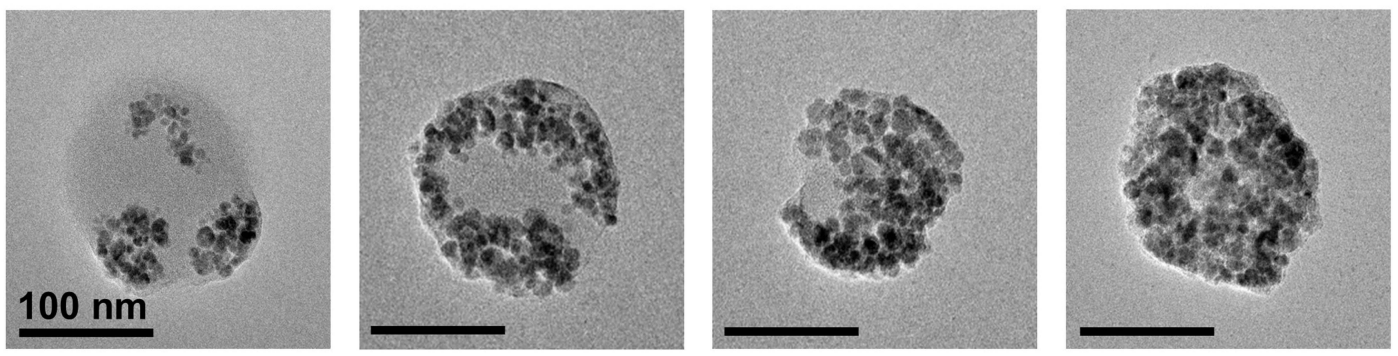

B

\section{C}
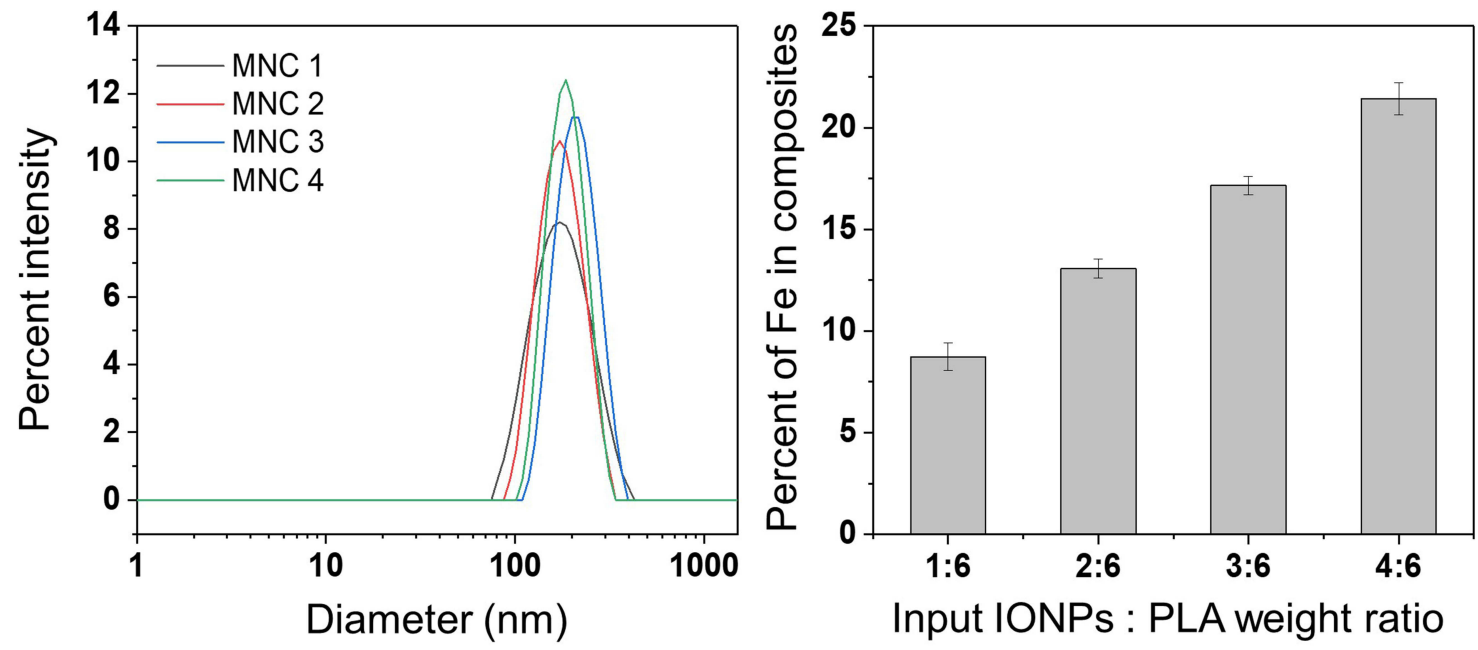

Figure 2 Characterization of the IONPs-embedded nanocomposites (MNCs). (A) TEM images of various MNCs. (B) Size distribution of MNCs. (C) Percent of Iron contents in MNCs $(n=3)$.

A

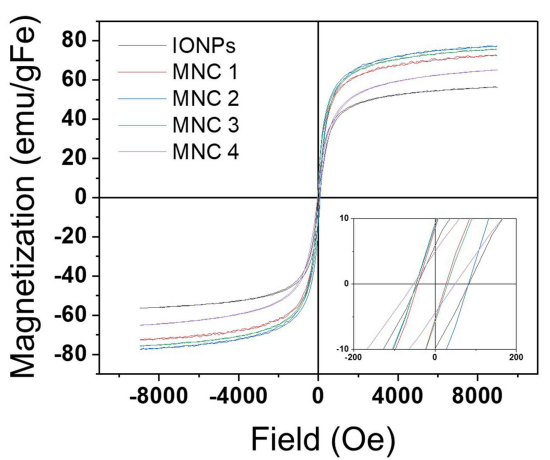

B

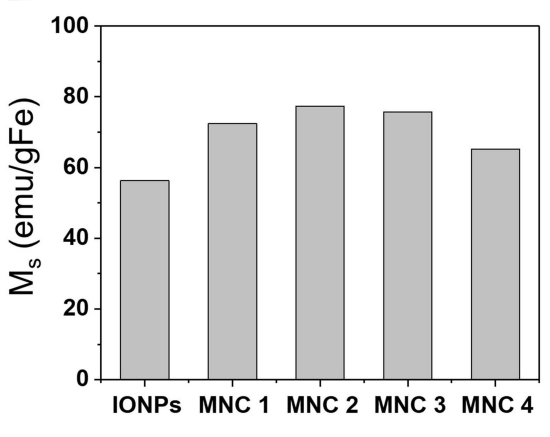

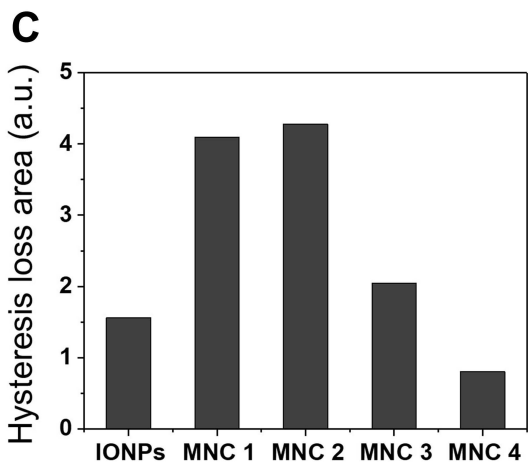

Figure 3 Magnetic properties of IONPs and various MNCs. (A) Magnetization of IONPs and MNCs. (B) Saturation magnetization (M) and (C) Hysteresis loss areas of IONPs and MNCs.

samples. The hysteresis loss areas decreased as the IONP content increased further in MNCs (Figure 3C).

\section{Magnetic Hyperthermia}

The hyperthermia properties of IONPs and MNCs were studied using an AMF $(H=3.25 \mathrm{kA} / \mathrm{m}$ and $f=191 \mathrm{kHz})$. The product of the field parameters $\left(H \times f=0.62 \times 10^{9}\right.$
$A m^{-1} s^{-1}$ ) was approximately 8 times less than the biological safety limit $\left(H \times f=5 \times 10^{9} \mathrm{Am}^{-1} \mathrm{~s}^{-1}\right){ }^{40}$ The heating performance of hydrophobic IONPs and various MNCs was measured in 1-octanol at an identical IONP concentration $(1 \mathrm{mg} \mathrm{Fe} / \mathrm{mL})$ and compared in terms of SLP. The SLP values of all MNCs were approximately two times larger than those of the nonembedded IONPs (Figures 4A 

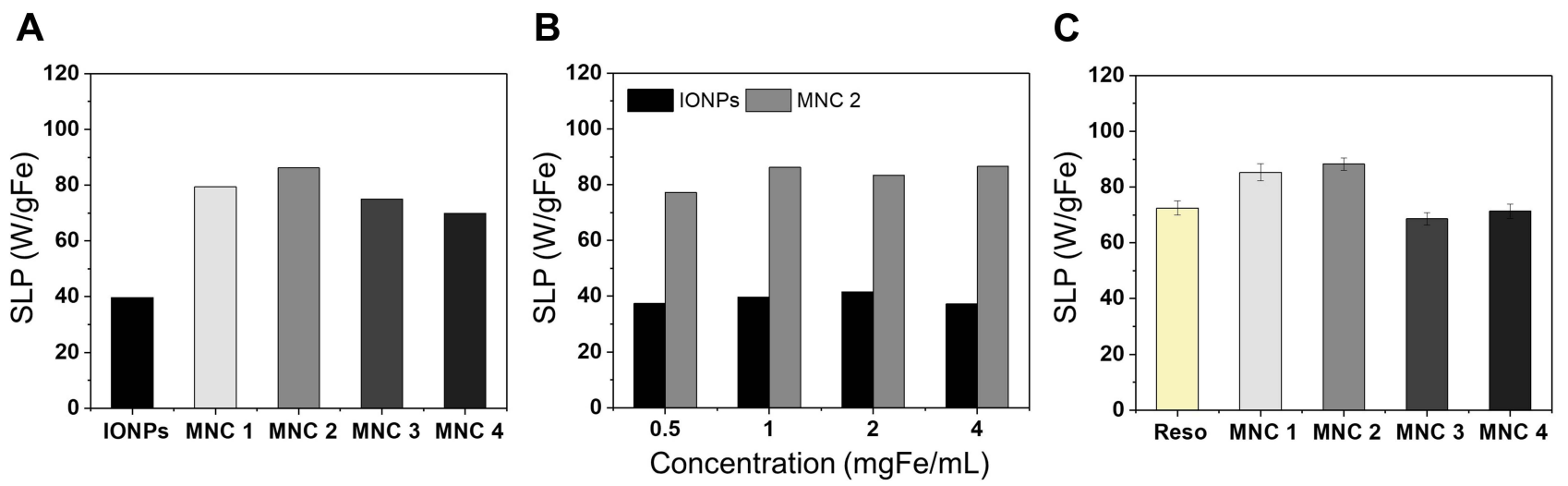

Figure 4 Heating efficiency of IONPs and MNCs. Heating efficiency was demonstrated as SLP values per gFe. (A) SLP values of IONPs and various MNCs at I mgFe/mL concentration in I-octanol. (B) SLP values of IONPs and MNC2 at different concentrations in I-octanol. (C) SLP of MNCs and Resovist ${ }^{\circledR}$ at 4 mgFe/mL concentration in aqueous solution.

and $\underline{\mathrm{S}}$ ). The results suggest that the SLP could be improved by embedding IONPs in a PLA polymer matrix, in which individual IONPs were close to others. Among the MNCs, MNC2 had the highest SLP value, which was similar to the results of the saturation magnetization and hysteresis area (Figure 3). For both IONPs and MNC2, heat generation efficiencies were not substantially affected by their concentrations between 0.5 and $4 \mathrm{mgFe} / \mathrm{mL}$ (Figure 4B), which implies that no substantial agglomeration of either IONPs or MNC2 occurred during the AMF application and SLP measurement. The SLP of the MNCs was further compared with that of commercial IONPs (ie, Resovist $^{\circledR}$ ) in an aqueous medium (DIW) (Figure 4C). The SLP values of MNC1 and MNC2 were higher than those of Resovist ${ }^{\circledR}$. Particularly, the SLP value of MNC2 was approximately $20 \%$ higher than that of Resovist ${ }^{\circledR}$ (Figure S5), implying the suitable performance of our MNCs for hyperthermia application.

The improved heat dissipation of MNCs is likely attributable to several possible factors. First, the high magnetic susceptibility (magnetization strength) of the MNCs might result in a high heating efficiency, based on the linear response theory. ${ }^{30}$ In the case of our MNC system, the IONPs are immobilized in the polymer matrix; hence, the Néel relaxation would play a major role in heat dissipation of the MNCs. In addition, as the IONP content increases, dipolar interactions would become substantial. Because the IONPs in the composites can behave collectively, such interparticle interactions may increase or decrease the anisotropy energy and heat dissipation depending on the nanoparticle composition, size, and homogeneity. ${ }^{53}$ Interparticle dipolar interactions generate a dipolar magnetic field and can increase the effective magnetic anisotropy, resulting in an increase in heat generation. Hysteresis loss can be a substantial heat generation mechanism for highly interactive magnetic nanomaterials. We observed that the hysteresis loss areas were higher for MNCs than for bare IONPs. In addition, at specific IONP contents (MNC1 and MNC2) such hysteresis loss was distinct (Figure 3D). It is also possible that the aggregation of individual IONPs in the PLA matrix resulted in a multidomain-like property and, thus, improved the hysteresis loss energy of the MNCs. However, excessive interparticle interactions might lead to decreases in magnetic susceptibility and hysteresis loss, resulting in a decrease in SLP. ${ }^{30,54,55}$ Dipolar interactions can cause magnetic nanoparticles stable against field orientation and increase the relaxation time. ${ }^{56,57}$ Several studies have reported the nonmonotonous heating performance of magnetic nanoparticles with different concentrations, indicating the optimal particle concentration for interparticle interactions and hyperthermic heat generation. ${ }^{35,58,59}$

\section{Effects of Magnetic Field Intensity and Frequency}

We further investigated the effects of AMF conditions (amplitude $(H)$ and frequency $(f)$ ) on heat generation by MNCs. Generally, the application of AMF with a higher $H$ or $f$ results in a higher SLP, and their influence on heat generation is different depending on the characteristics of the magnetic nanoparticles. ${ }^{15}$ We first measured the SLP of the samples by varying the amplitudes $(H=1.6 \sim 3.2$ $\mathrm{kA} / \mathrm{m}$ ) with constant frequency $(f=191 \mathrm{kHz}$ ) (Figure $5 \mathrm{~A}$ ). All nanocomposites showed linear increases in SLP as the 
A

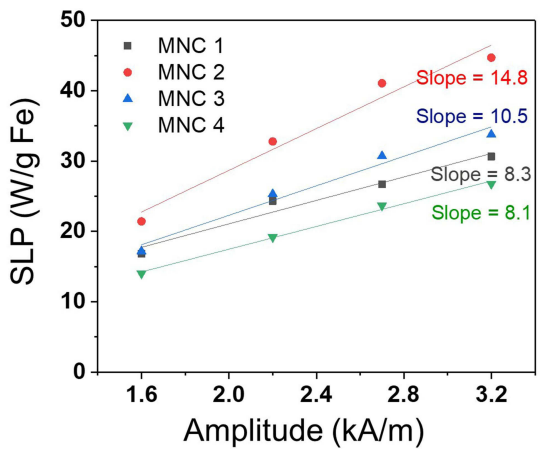

B

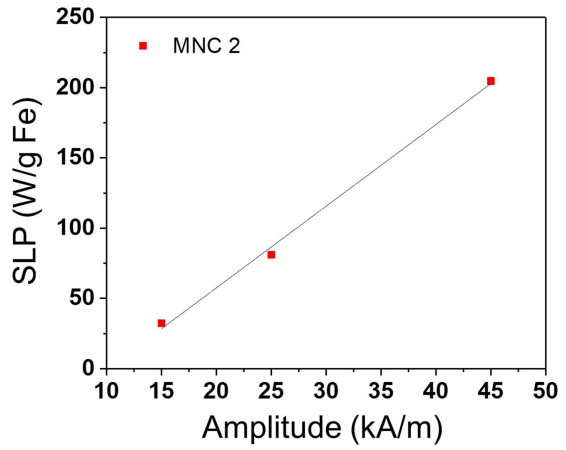

C

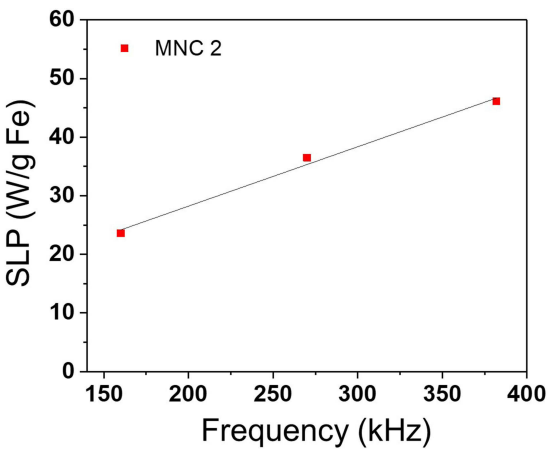

Figure 5 Effects of AMF on heating efficiency of MNCs. (A) Influence of amplitude ( $1.6-3.2 \mathrm{kA} / \mathrm{m}$ ) on heat generation of various MNCs. (B) Influence of the amplitude (I5$45 \mathrm{kA} / \mathrm{m})$ on heat generation of MNC2. (C) Influence of frequency $(160-382 \mathrm{kHz})$ on heat generation of MNC2. All experiments were performed at the sample IONP concentration (I mgFe/mL).

amplitude increased. A two-fold increase in the amplitude resulted in increases in the SLP values by 1.8-, 2.1-, 2.0-, and 1.9-fold for MNC1, MNC2, MNC3, and MNC4, respectively. In addition, in the amplitude-SLP plots, MNC2 showed the highest slope among the samples. Accordingly, MNC2 was further analyzed to study the effects of different $H$ and $f$ ranges on SLP. Specifically, we applied a wide and high range of AMF amplitude (15$45 \mathrm{kA} / \mathrm{m}$ ) using a lab-made AMF instrument (Figure 5B). MNC2 exhibited a linear relationship between SLP and an applied amplitude, with a drastic increase in SLP as the amplitude increased. For example, SLP values of MNC2 were 32.2, 81.1, and 204.7 (W/gFe) under AMF application of 15,25 , and $45 \mathrm{kA} / \mathrm{m}$, respectively. In addition, MNC2 exhibited a linear relationship between the SLP and $\mathrm{AMF}$ frequency (Figure 5C). The SLP values were 23.6, 36.5, and 46.1 (W/gFe) with AMF of 160, 270, and $382 \mathrm{kHz}$, respectively. An increase in the frequency $(2.4$ times) resulted in a 2 -fold increase in SLP. The results revealed that the influence of the amplitude on the heat generation of MNC2 was substantially greater than that of frequency. We could obtain the highest SLP value (204.7 $\mathrm{W} / \mathrm{gFe}$ ) from MNC2 with $H=45 \mathrm{kA} / \mathrm{m}$ and $f=97 \mathrm{kHz}$ $\left(H \times f=4.4 \times 10^{9} \mathrm{Am}^{-1} \mathrm{~s}^{-1}\right)$, which is below the biosafety limit.

\section{In vitro Colloidal Stability}

The stability of magnetic nanoparticles in the physiological environment is essential for their robust and reliable biomedical applications because many magnetic nanoparticles, including IONPs, undergo severe agglomeration in practical applications, such as in vivo conditions. For stability tests, MNC2, which showed the highest heat generation efficiency among the samples, was selected and compared with Resovist ${ }^{\circledR}$. After incubation in aqueous solutions, both samples appeared to remain stable without severe aggregation or color changes (Figure 6A). The hydrodynamic size of MNC2 remained unchanged after incubation in both PBS and DMEM. In contrast, the size of Resovist ${ }^{\circledR}$ gradually increased in DMEM, almost doubling after $48 \mathrm{~h}$ in DMEM (Figure 6B). In addition, the SLP value of MNC2 did not significantly change after 48 $\mathrm{h}$ incubation in DMEM, whereas the SLP value of Resovist $^{\circledR}$ decreased by $14 \%$ (Figure $6 \mathrm{C}$ ). These results indicate that $\mathrm{MNC2}$ is stable in physiological environments. Such excellent stability of MNC2 may be attributed to the stable PLA matrix, which physically prevents direct interactions of the individual IONPs and their spontaneous aggregation. In addition, PLA presents a mild surface charge (zeta potential is -0.4 to -1 in physiological conditions), which offers stability in aqueous solutions. ${ }^{60}$ Conversely, the aggregation of Resovist ${ }^{\circledR}$ led to a change in interparticle interactions and performance deterioration. $^{50}$

\section{Cytocompatibility Tests}

The cytocompatibility of MNC2 and Resovist ${ }^{\circledR}$ was examined by in vitro culture of NIH-3T3 fibroblasts (Figure 7). The cell viability cultured with $\mathrm{MNC} 2$ remained high ( $>$ $96 \%$ ) - up to $2 \mathrm{mgFe} / \mathrm{mL}$ concentration — corresponding to $15.4 \mathrm{mg}$ MNC2 per mL medium, after $1 \mathrm{~d}$ in culture. In contrast, the viability of Resovist ${ }^{\circledR}$ gradually decreased with an increase in its concentration in the culture medium. For example, the cell viability in Resovist ${ }^{\circledR}$ sample was $>90 \%$ up to $0.5 \mathrm{mgFe} / \mathrm{mL}$; however, at a higher concentration, the cell viability significantly decreased. 
A

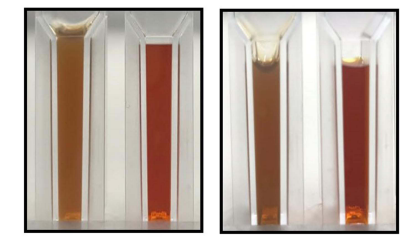

$\mathbf{O h}$ In DMEM

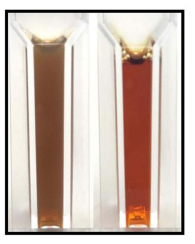

$\mathbf{O h}$

\section{B}

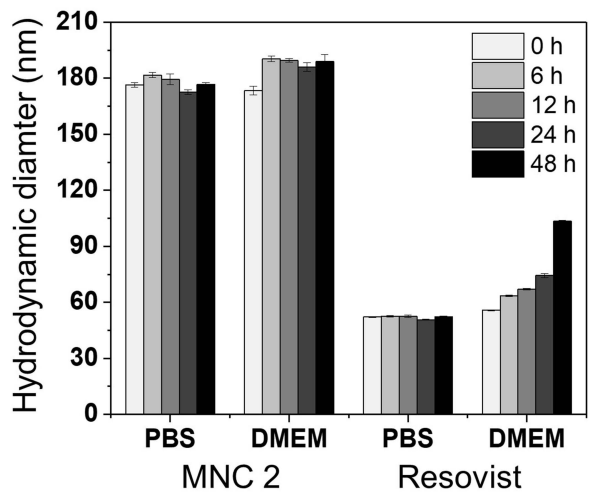

C

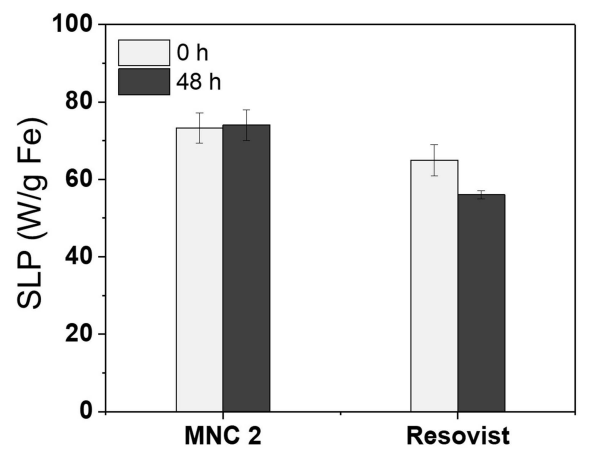

Figure 6 Stability tests of MNC2 and Resovist ${ }^{\circledR}$. (A) Photographs of the MNC2 (left) and Resovist ${ }^{\circledR}$ (right) during the incubation in PBS or DMEM. (B) Hydrodynamic diameters of MNC2 and Resovist ${ }^{\circledR}$ during the incubation in PBS and media for $48 \mathrm{~h}$. (C) SLP values before and after the $48 \mathrm{~h}$ incubation in DMEM medium.

A
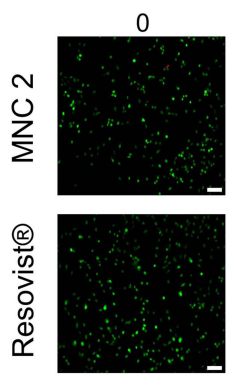

$m g \mathrm{Fe} / \mathrm{mL}$
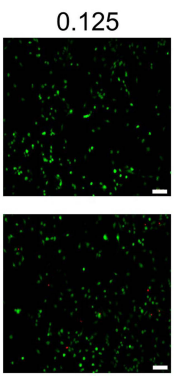
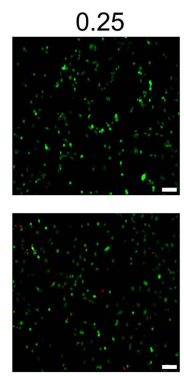
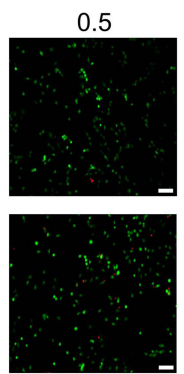
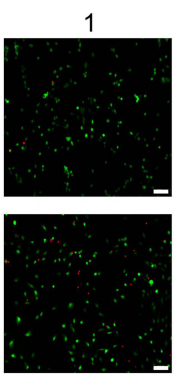

B

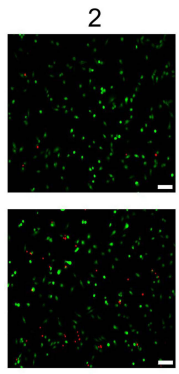

Figure 7 Cytocompatibility tests of the MNC2 and Resovist ${ }^{\circledR}$ with NIH-3T3 cells. (A) Fluorescence micrographs of the cells after the incubation with MNC2 and Resovist ${ }^{\circledR}$ at different concentrations for I d. The green and red cells in the images indicate live and dead cells, respectively. Scale bars are $100 \mu \mathrm{m}$. (B) Percent viability of the cells cultured with various samples for I d. An asterisk *Indicates the statistical significance between two groups $(p<0.05)$.

For example, the cell viability of Resovist ${ }^{\circledR}$ at $2 \mathrm{mg} \mathrm{Fe} / \mathrm{mL}$ was $77 \%$. Resovist ${ }^{\circledR}$ is a carboxydextran-coated IONP with hydroxyl groups on the surface. ${ }^{61}$ Different nanoparticles exhibit different cellular uptake, distribution, and reactivity (eg, reactive oxygen species formation) depending on their size, shape, and surface characteristics, which can affect toxicity. ${ }^{62}$ We speculate that the relative toxicity of Resovist ${ }^{\circledR}$ might be attributed to its large surface area and instability, resulting in particle aggregation. ${ }^{63}$ As stated earlier, the materials used in this study (ie, IONPs and PLA) are all biodegradable and biocompatible; hence, MNCs are believed to be minimally toxic (Figure S6).${ }^{1-}$ ${ }^{3,41,64}$ In addition, the excellent stability of MNCs may relieve a concern with the potential toxicity associated with particle instability (eg, aggregation).

\section{In vivo Biodistribution and Toxicity Study} The Cy7-loaded MNC was intravenously injected via tail vein to evaluate biodistribution. In vivo biodistribution of $\mathrm{MNC}$ was detected at the time points of $0,4,8,12,24$, and $48 \mathrm{~h}$ of post-injection. According to in vivo biodistribution images (Figure 8A), the injected MNC appeared to first circulate in the whole body through bloodstream and trap in specific organs of the body. In addition, we observed overall decreases in fluorescence signals $4 \mathrm{~h}$ after the injection (Figure 8A). As shown in Figure 8B, noticeable signals were observed from liver, spleen, and heart tissues post injection while minimal intensity was detected in the lung at both 24 and $48 \mathrm{~h}$ after injection. In general, the major fraction of the intravenously injected IONPs is first filtered by the liver and spleen, and reaches other organs. ${ }^{65}$ Based on the fluorescence intensity, the 
A

0

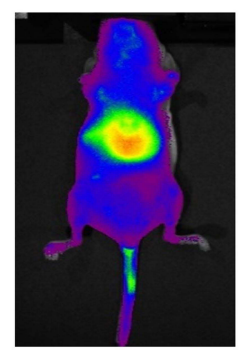

B

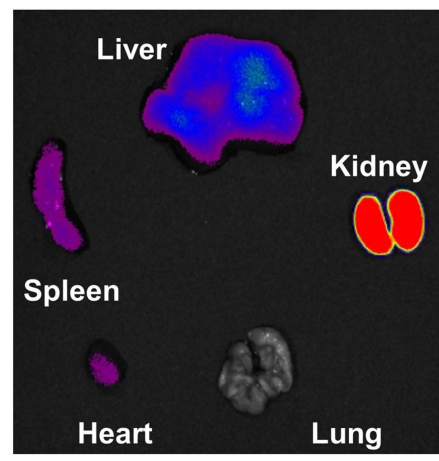

4

$24 \mathrm{~h}$
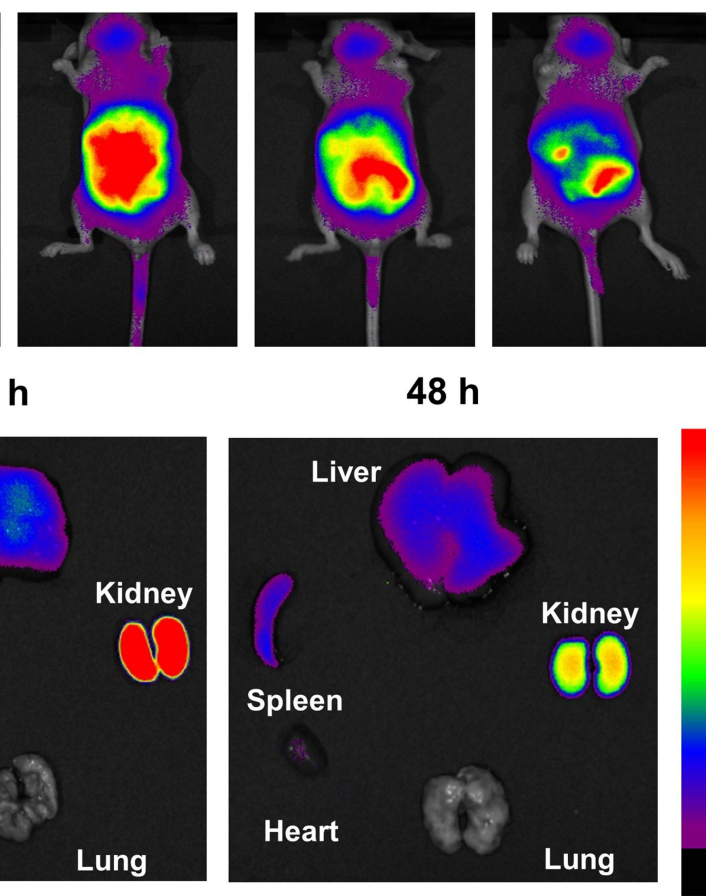

hour

8

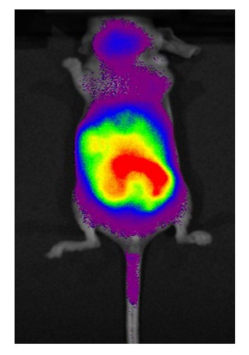

$48 \mathrm{~h}$
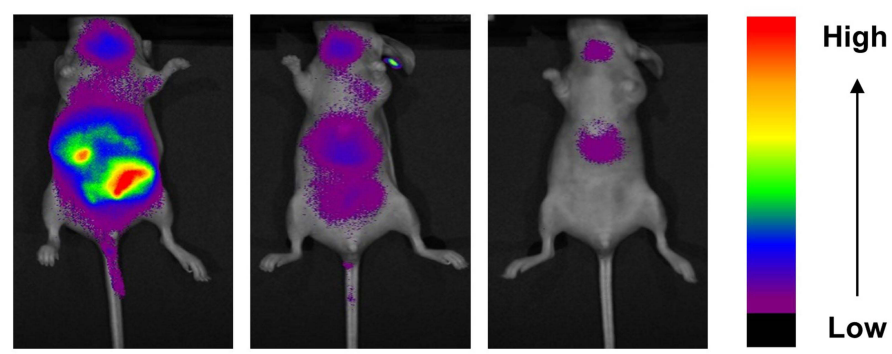

C

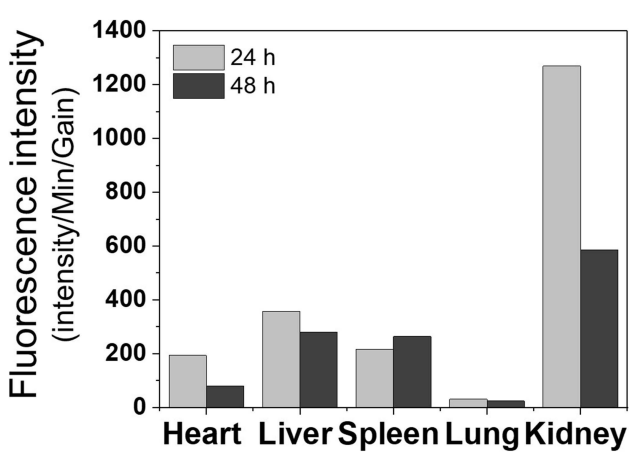

Figure 8 Biodistribution of MNC after intravenous injection. (A) In vivo fluorescence images of the mice acquired after 0, 4, 8, I2, 24, and 48 h of MNC injection. (B) Fluorescence images of the main organs after $24 \mathrm{~h}$ and $48 \mathrm{~h}$ of injection. (C) Fluorescence intensities of the individual organs harvested at $24 \mathrm{~h}$ and $48 \mathrm{~h}$ after injection of MNC.

relative amount of the MNC accumulated in the individual organs were compared. Fluorescence intensities of the most organs except spleen slightly decreased after $48 \mathrm{~h}$ of MNC injection, implying the MNC might slowly degrade or be excreted (Figure 8C). Mononuclear phagocytic systems (MPS), such as liver, might play roles in degrading IONPs in the blood. ${ }^{66}$ For example, Kupffer cells in the liver are known to actively degrade the IONPs. ${ }^{65}$
After administration of the MNC, all the mice behaved normally over the entire observation period, indicating that the MNC did not induce apparent toxicity in vivo. Furthermore, several organs were harvested at $24 \mathrm{~h}$ and $48 \mathrm{~h}$ after the injection of control (sham) and MNC for histological analysis (Figure 9). H\&E staining of the organs revealed that the heart, liver, spleen, lung and kidney showed no obvious difference between the untreated control and the MNC treated groups
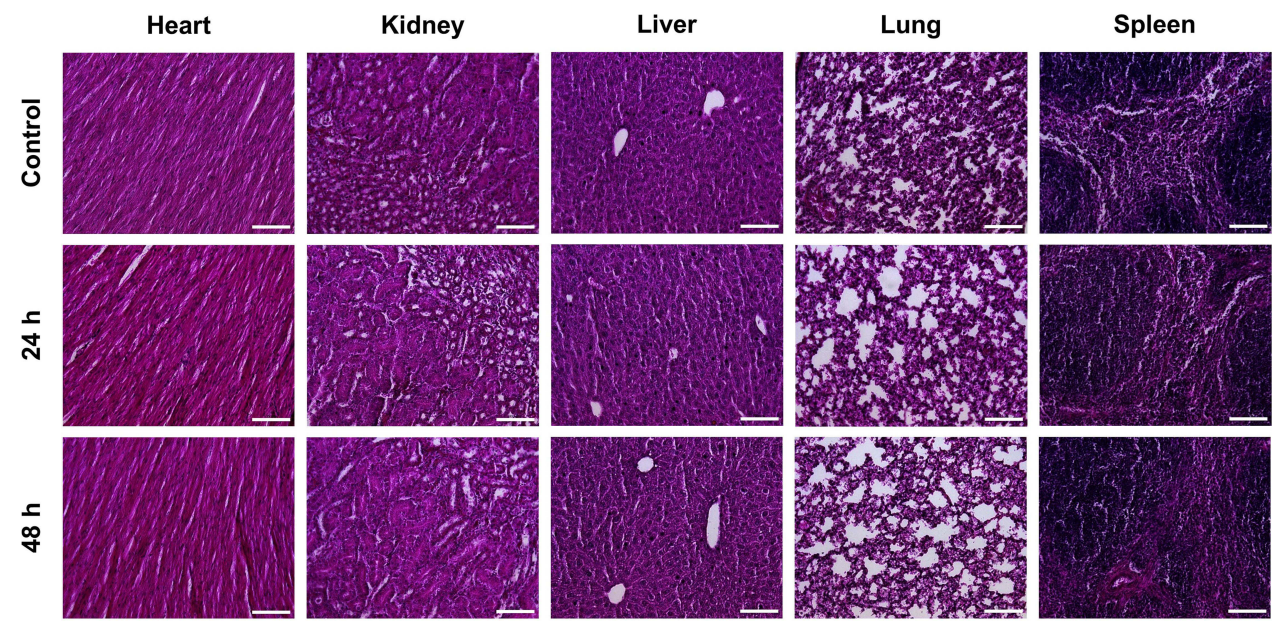

Figure 9 Histological images of main organs (heart, kidney, liver, lung, and spleen) after $24 \mathrm{~h}$ and $48 \mathrm{~h}$ of MNC injection and the untreated control group. Scale bar $=100 \mu \mathrm{m}$. 
with respect to the characteristic tissue structures. Therefore, MNC was determined to be biocompatible.

\section{Conclusion}

To produce stable and high-performance magnetic nanomaterials for potential hyperthermia applications, we synthesized magnetic nanocomposites by embedding IONPs in PLA while controlling the IONP content in the composites. The fabricated MNCs showed enhanced magnetization and heating efficiency compared with nonembedded IONPs. Notably, MNC2 containing moderate amounts of IONPs (13 wt $\%$ of Fe) showed higher magnetization and heating efficiency (ie, SLP) than the other nanocomposites. Further, these maximized characteristics appear to originate from the favorable interparticle interactions. In addition, MNC2 exhibited excellent colloidal stability in aqueous solutions, as evidenced by negligible changes in hydrodynamic sizes and SLP after the incubation, which led to consistent heating in physiological environments against the clinically approved IONPs, Resovist ${ }^{\circledR}$. In vitro cell cytotoxicity tests revealed that MNC2 had good cytocompatibility-superior to Resovist ${ }^{\circledR}$. In vivo study indicated that MNC had great biocompatibility. Altogether, we successfully produce MNCs displaying improved magnetic properties, heating performance, stability, and biocompatibility. These unique properties will make our MNCs have great potential for various hyperthermia applications. In addition, our MNCs were prepared using widely used biocompatible materials (ie, PLA and IONPs) for their potential translation to clinical applications. Nevertheless, future studies, such as in vivo applications for cancer treatment, are required to clearly demonstrate their efficacy and biocompatibility.

\section{Acknowledgments}

This work was supported by grants of the National Research Foundation of Korea (NRF) funded by the Ministry of Science, ICT and Future Planning (NRF2019M3C1B8090799 and NRF- 2021R1A4A3025206).

\section{Disclosure}

Professor Jae Young Lee reports a patent KR20210016924 pending. The authors report no other conflicts of interest in this work.

\section{References}

1. Wu W, Wu Z, Yu T, Jiang C, Kim WS. Recent progress on magnetic iron oxide nanoparticles: synthesis, surface functional strategies and biomedical applications. Sci Technol Adv Mater. 2015;16(2):023501. doi:10.1088/1468-6996/16/2/023501

2. Sangaiya P, Jayaprakash R. A review on iron oxide nanoparticles and their biomedical applications. J Supercond Nov Magn. 2018;31 (11):3397-3413. doi:10.1007/s10948-018-4841-2

3. Prashant C, Dipak M, Yang CT, Chuang KH, Jun D, Feng SS. Superparamagnetic iron oxide - loaded poly (lactic acid)-d- $\alpha-$ tocopherol polyethylene glycol 1000 succinate copolymer nanoparticles as MRI contrast agent. Biomaterials. 2010;31(21):5588-5597. doi:10.1016/j.biomaterials.2010.03.070

4. Pouponneau P, Leroux JC, Soulez G, Gaboury L, Martel S. Coencapsulation of magnetic nanoparticles and doxorubicin into biodegradable microcarriers for deep tissue targeting by vascular MRI navigation. Biomaterials. 2011;32(13):3481-3486. doi:10.1016/j. biomaterials.2010.12.059

5. Sivakumar B, Aswathy RG, Romero-Aburto R, et al. Highly versatile SPION encapsulated PLGA nanoparticles as photothermal ablators of cancer cells and as multimodal imaging agents. Biomater Sci. 2017;5 (3):432-443. doi:10.1039/C6BM00621C

6. Hayashi K, Nakamura M, Sakamoto W, et al. Superparamagnetic nanoparticle clusters for cancer theranostics combining magnetic resonance imaging and hyperthermia treatment. Theranostics. 2013;3(6):366.

7. Zhang ZQ, Song SC. Multiple hyperthermia-mediated release of TRAIL/SPION nanocomplex from thermosensitive polymeric hydrogels for combination cancer therapy. Biomaterials. 2017;132:16-27. doi:10.1016/j.biomaterials.2017.03.049

8. Jeon H, Kim J, Lee YM, et al. Poly-paclitaxel/cyclodextrin-SPION nano-assembly for magnetically guided drug delivery system. J Controlled Release. 2016;231:68-76. doi:10.1016/j.jconrel.2016.01.006

9. Neuberger T, Schöpf B, Hofmann H, Hofmann M, Von Rechenberg B. Superparamagnetic nanoparticles for biomedical applications: possibilities and limitations of a new drug delivery system. J Magn Magn Mater. 2005;293(1):483-496. doi:10.1016/j. jmmm.2005.01.064

10. Veiseh O, Gunn JW, Zhang M. Design and fabrication of magnetic nanoparticles for targeted drug delivery and imaging. Adv Drug Deliv Rev. 2010;62(3):284-304. doi:10.1016/j.addr.2009.11.002

11. Chen R, Romero G, Christiansen MG, Mohr A, Anikeeva P. Wireless magnetothermal deep brain stimulation. Science. 2015;347 (6229):1477-1480. doi:10.1126/science.1261821

12. Tay A, Di Carlo D. Magnetic nanoparticle-based mechanical stimulation for restoration of mechano-sensitive ion channel equilibrium in neural networks. Nano Lett. 2017;17(2):886-892. doi:10.1021/acs. nanolett.6b04200

13. Munshi R, Qadri SM, Zhang Q, Castellanos Rubio I, Del Pino P, Pralle A. Magnetothermal genetic deep brain stimulation of motor behaviors in awake, freely moving mice. eLife. 2017;6:e27069. doi:10.7554/eLife. 27069

14. Lv Y, Yang Y, Fang J, et al. Size dependent magnetic hyperthermia of octahedral $\mathrm{Fe} \quad{ }_{3} \quad \mathrm{O}_{4}$ nanoparticles. RSC $A d v . \quad 2015 ; 5$ (94):76764-76771. doi:10.1039/C5RA12558H

15. Kandasamy G, Sudame A, Bhati P, Chakrabarty A, Kale SN, Maity D. Systematic magnetic fluid hyperthermia studies of carboxyl functionalized hydrophilic superparamagnetic iron oxide nanoparticles based ferrofluids. J Colloid Interface Sci. 2018;514:534-543. doi:10.1016/j.jcis.2017.12.064

16. Bae KH, Park M, Do MJ, et al. Chitosan oligosaccharide-stabilized ferrimagnetic iron oxide nanocubes for magnetically modulated cancer hyperthermia. ACS Nano. 2012;6(6):5266-5273. doi:10.1021/ nn301046w 
17. Cardoso VF, Francesko A, Ribeiro C, Bañobre-López M, Martins P, Lanceros-Mendez S. Advances in magnetic nanoparticles for biomedical applications. Adv Healthc Mater. 2018;7(5):1700845.

18. Li F, Lu J, Kong X, Hyeon T, Ling D. Dynamic nanoparticle assemblies for biomedical applications. Adv Mater. 2017;29(14):1605897. doi:10.1002/adma.201605897

19. Noh S, Moon SH, Shin TH, Lim Y, Cheon J. Recent advances of magneto-thermal capabilities of nanoparticles: from design principles to biomedical applications. Nano Today. 2017;13(61-76):61-76. doi:10.1016/j.nantod.2017.02.006

20. Branquinho LC, Carrião MS, Costa AS, et al. Effect of magnetic dipolar interactions on nanoparticle heating efficiency: implications for cancer hyperthermia. Sci Rep. 2013;3(1):2887. doi:10.1038/ srep02887

21. Tong S, Quinto CA, Zhang L, Mohindra P, Bao G. Size-dependent heating of magnetic iron oxide nanoparticles. ACS Nano. 2017;11 (7):6808-6816. doi:10.1021/acsnano.7b01762

22. Martinez-Boubeta C, Simeonidis K, Makridis A, et al. Learning from nature to improve the heat generation of iron-oxide nanoparticles for magnetic hyperthermia applications. Sci Rep. 2013;3(1):1652. doi:10.1038/srep01652

23. Fortin JP, Gazeau F, Wilhelm C. Intracellular heating of living cells through Néel relaxation of magnetic nanoparticles. Eur Biophys J. 2008;37(2):223-228. doi:10.1007/s00249-007-0197-4

24. Chung SH, Hoffmann A, Bader SD, et al. Biological sensors based on Brownian relaxation of magnetic nanoparticles. Appl Phys Lett. 2004;85(14):2971-2973. doi:10.1063/1.1801687

25. Wang D, Lin B, Shen T, et al. The effect of neighbor distance of magnetic nanoparticle clusters on magnetic resonance relaxation properties. Sci Bull. 2016;61(13):1023-1030. doi:10.1007/s11434-016-1107-x

26. Nguyen TDT, Pitchaimani A, Ferrel C, Thakkar R, Aryal S. Nanoconfinement-driven enhanced magnetic relaxivity of SPIONs for targeted tumor bioimaging. Nanoscale. 2018;11:284-294.

27. Wang D, Lin B, Shen T, et al. Control of the interparticle spacing in superparamagnetic iron oxide nanoparticle clusters by surface ligand engineering. Chin Phy B. 2016;25(7):9.

28. Hayashi K, Sato Y, Sakamoto W, Yogo T. Theranostic nanoparticles for MRI-guided thermochemotherapy: "tight" clustering of magnetic nanoparticles boosts relaxivity and heat-generation power. ACS Biomater Sci Eng. 2017;3(1):95-105. doi:10.1021/acsbiomaterials.6b00536

29. Pöselt E, Kloust H, Tromsdorf U, et al. Relaxivity optimization of a PEGylated iron-oxide-based negative magnetic resonance contrast agent for $T_{2}$-weighted spin-echo imaging. ACS Nano. 2012;6 (2):1619-1624. doi:10.1021/nn204591r

30. Sakellari D. Ferrimagnetic nanocrystal assemblies as versatile magnetic particle hyperthermia mediators. Mater Sci Eng C. 2016;7:187-193.

31. Sadat ME, Patel R, Sookoor J, et al. Effect of spatial confinement on magnetic hyperthermia via dipolar interactions in Fe3O4 nanoparticles for biomedical applications. Mater Sci Eng C. 2014;42:52-63. doi:10.1016/j.msec.2014.04.064

32. Coral DF, Mendoza Zélis P, Marciello M, et al. Effect of nanoclustering and dipolar interactions in heat generation for magnetic hyperthermia. Langmuir. 2016;32(5):1201-1213. doi:10.1021/acs. langmuir.5b03559

33. Jeun M, Bae S, Tomitaka A, et al. Effects of particle dipole interaction on the ac magnetically induced heating characteristics of ferrite nanoparticles for hyperthermia. Appl Phys Lett. 2009;95(8):082501. doi:10.1063/1.3211120

34. Cai Z, Wu C, Yang L, Wang D, Ai H. Assembly-controlled magnetic nanoparticle clusters as MRI contrast agents. ACS Biomater Sci Eng. 2020;6(5):2533-2542. doi:10.1021/acsbiomaterials.9b01198

35. Evans BA, Bausch MD, Sienerth KD, Davern MJ. Non-monotonicity in the influence of nanoparticle concentration on SAR in magnetic nanoparticle hyperthermia. J Magn Magn Mater. 2018;465:559-565. doi:10.1016/j.jmmm.2018.06.051
36. Etheridge ML, Hurley KR, Zhang J, et al. Accounting for biological aggregation in heating and imaging of magnetic nanoparticles. TECHNOLOGY. 2014;02(03):214-228. doi:10.1142/ S2339547814500198

37. Hurley KR, Ring HL, Etheridge M, et al. Predictable heating and positive MRI contrast from a mesoporous silica-coated iron oxide nanoparticle. Mol Pharm. 2016;13(7):2172-2183. doi:10.1021/acs. molpharmaceut.5b00866

38. Eberbeck D, Wiekhorst F, Steinhoff U, Trahms L. Aggregation behaviour of magnetic nanoparticle suspensions investigated by magnetorelaxometry. J Phys Condens Matter. 2006;18(38):S2829_ S2846. doi:10.1088/0953-8984/18/38/S20

39. Hergt R, Dutz S. Magnetic particle hyperthermia-biophysical limitations of a visionary tumour therapy. J Magn Magn Mater. 2007;311(1):187-192. doi:10.1016/j.jmmm.2006.10.1156

40. Guardia P, Di Corato R, Lartigue L, et al. Water-soluble iron oxide nanocubes with high values of specific absorption rate for cancer cell hyperthermia treatment. ACS Nano. 2012;6(4):3080-3091. doi:10.1021/nn2048137

41. Thirunavukkarasu GK, Cherukula K, Lee H, Jeong YY, Park IK, Lee JY. Magnetic field-inducible drug-eluting nanoparticles for image-guided thermo-chemotherapy. Biomaterials. 2018;18 0:240-252. doi:10.1016/j.biomaterials.2018.07.028

42. Lee H, Thirunavukkarasu GK, Kim S, Lee JY. Remote induction of in situ hydrogelation in a deep tissue, using an alternating magnetic field and superparamagnetic nanoparticles. Nano Res. 2018;11 (11):5997-6009. doi:10.1007/s12274-018-2114-9

43. Hergt R, Dutz S, Röder M. Effects of size distribution on hysteresis losses of magnetic nanoparticles for hyperthermia. J Phys Condens Matter. 2008;20(38):385214. doi:10.1088/0953-8984/20/38/385214

44. Darwish MS, Kim H, Lee H, Ryu C, Lee JY, Yoon J. Synthesis of magnetic ferrite nanoparticles with high hyperthermia performance via a controlled co-precipitation method. Nanomaterials. 2019;9 (8):1176. doi:10.3390/nano9081176

45. Cardiff RD, Miller CH, Munn RJ. Manual hematoxylin and eosin staining of mouse tissue sections. Cold Spring Harb Protoc. 2014;2014(6):pdb.prot073411-pdb.prot073411. doi:10.1101/pdb. prot073411

46. Hugounenq P, Levy M, Alloyeau D, et al. Iron oxide monocrystalline nanoflowers for highly efficient magnetic hyperthermia. J Phys Chem C. 2012;116(29):15702-15712. doi:10.1021/jp3025478

47. Urtizberea A, Natividad E, Arizaga A, Castro M, Mediano A. Specific absorption rates and magnetic properties of ferrofluids with interaction effects at low concentrations. J Phys Chem C. 2010;114 (11):4916-4922. doi:10.1021/jp912076f

48. de la Presa P, Luengo Y, Velasco V, et al. Particle interactions in liquid magnetic colloids by zero field cooled measurements: effects on heating efficiency. J Phys Chem C. 2015;119(20):11022-11030. doi:10.1021/jp5115515

49. Muscas G. The interplay between single particle anisotropy and interparticle interactions in ensembles of magnetic nanoparticles. Phys Chem Chem Phys. 2018;20:28634-28643.

50. Mohapatra J, Xing M, Liu JP. Inductive thermal effect of ferrite magnetic nanoparticles. Materials. 2019;12(19):3208. doi:10.3390/ ma12193208

51. Santos PJ, Macfarlane RJ. Reinforcing supramolecular bonding with magnetic dipole interactions to assemble dynamic nanoparticle superlattices. J Am Chem Soc. 2020;142(3):1170-1174. doi:10.1021/jacs.9b11476

52. Abu-Bakr AF, Zubarev A. Effect of interparticle interaction on magnetic hyperthermia: homogeneous spatial distribution of the particles. Philos Trans R Soc Math Phys Eng Sci. 2019;377(2143):20180216.

53. Abenojar EC, Wickramasinghe S, Bas-Concepcion J, Samia ACS. Structural effects on the magnetic hyperthermia properties of iron oxide nanoparticles. Prog Nat Sci Mater Int. 2016;26(5):440-448. doi:10.1016/j.pnsc.2016.09.004 
54. Serantes D, Baldomir D, Martinez-Boubeta C, et al. Influence of dipolar interactions on hyperthermia properties of ferromagnetic particles. J Appl Phys. 2010;108(7):073918. doi:10.1063/1.3488881

55. Materia ME, Guardia P, Sathya A, et al. Mesoscale assemblies of iron oxide nanocubes as heat mediators and image contrast agents. Langmuir. 2015;31(2):808-816. doi:10.1021/la503930s

56. Conde-Leboran I, Baldomir D, Martinez-Boubeta C, et al. A single picture explains diversity of hyperthermia response of magnetic nanoparticles. J Phys Chem C. 2015;119(27):15698-15706. doi:10.1021/acs.jpcc.5b02555

57. Salas G, Camarero J, Cabrera D, et al. Modulation of magnetic heating via dipolar magnetic interactions in monodisperse and crystalline iron oxide nanoparticles. J Phys Chem C. 2014;118 (34):19985-19994. doi:10.1021/jp5041234

58. Martinez-Boubeta C, Simeonidis K, Serantes D, et al. Adjustable hyperthermia response of self-assembled ferromagnetic $\mathrm{Fe}-\mathrm{MgO}$ core-shell nanoparticles by tuning dipole-dipole interactions. $A d v$ Funct Mater. 2012;22(17):3737-3744. doi:10.1002/adfm.201200307

59. Diamantopoulos G, Basina G, Tzitzios V, et al. Magnetic hyperthermia of laponite based ferrofluid. $J$ Magn Magn Mater. 2013;336:71-74. doi:10.1016/j.jmmm.2013.02.032

60. Da Silva J, Jesus S, Bernardi N, Colaço M, Borges O. Poly(D, L-Lactic Acid) nanoparticle size reduction increases its immunotoxicity. Front Bioeng Biotechnol. 2019;7:137. doi:10.3389/ fbioe.2019.00137
61. Abdollah MRA, Kalber T, Tolner B, et al. Prolonging the circulatory retention of SPIONs using dextran sulfate: in vivo tracking achieved by functionalisation with near-infrared dyes. Faraday Discuss. 2014;175:41-58. doi:10.1039/C4FD00114A

62. Hayman I, Mehl P, Kapoor V, Wilson O. Toxicity of BSA-stabilized silver nanoparticles on immune circulating cells. MRS Proc. 2007;1061:1061-MM09-18. doi:10.1557/PROC-1061-MM09-18

63. Rabolli V, Thomassen LCJ, Uwambayinema F, Martens JA, Lison D. The cytotoxic activity of amorphous silica nanoparticles is mainly influenced by surface area and not by aggregation. Toxicol Lett. 2011;206(2):197-203. doi:10.1016/j.toxlet.2011.07.013

64. Chen CC, Chueh JY, Tseng H, Huang HM, Lee SY. Preparation and characterization of biodegradable PLA polymeric blends. Biomaterials. 2003;24(7):1167-1173. doi:10.1016/S0142-9612(02) 00466-0

65. Arami H, Khandhar A, Liggitt D, Krishnan KM. In vivo delivery, pharmacokinetics, biodistribution and toxicity of iron oxide nanoparticles. Chem Soc Rev. 2015;44(23):8576-8607. doi:10.1039/ c5cs00541h

66. Lee MJE, Veiseh O, Bhattarai N, et al. Rapid pharmacokinetic and biodistribution studies using cholorotoxin-conjugated iron oxide nanoparticles: a novel non-radioactive method. PLoS One. 2010;5 (3):e9536. doi:10.1371/journal.pone.0009536
International Journal of Nanomedicine

\section{Publish your work in this journal}

The International Journal of Nanomedicine is an international, peerreviewed journal focusing on the application of nanotechnology in diagnostics, therapeutics, and drug delivery systems throughout the biomedical field. This journal is indexed on PubMed Central, MedLine, CAS, SciSearch ${ }^{\mathbb{}}$, Current Contents ${ }^{\mathbb{R}} /$ Clinical Medicine, $^{2}$

\section{Dovepress}

Journal Citation Reports/Science Edition, EMBase, Scopus and the Elsevier Bibliographic databases. The manuscript management system is completely online and includes a very quick and fair peer-review system, which is all easy to use. Visit http://www.dovepress.com/ testimonials.php to read real quotes from published authors. 